\title{
Unusual Presentation of Cerebral Lupus: A Case Report of Parkinsonism in Cerebral Lupus
}

\author{
Chee Keong Chang Malehah Mohd Noh Constance Liew Sat Lin \\ Alvin Oliver Payus \\ Faculty of Medicine and Health Science, Universiti Malaysia Sabah (UMS), Kota Kinabalu, \\ Malaysia
}

\section{Keywords}

Systemic lupus erythematosus $\cdot$ Parkinsonism $\cdot$ Cerebral lupus

\begin{abstract}
Systemic lupus erythematosus (SLE) is a chronic autoimmune disease with multisystem involvement that follows a relapsing and remitting course. It is characterized by an immunemediated response to own body defense mechanism and mistakenly attacked healthy cells of the skin, joints, kidneys, blood cells, and nervous system. Cerebral lupus refers to a constellation of neurological and/or behavioral clinical syndromes in patients with SLE. The spectrum of presentation can vary widely ranging from mild symptoms such as headaches, slight cognitive dysfunction, and mood disorders to more serious conditions like seizures, stroke, or coma. This case report is about a case of cerebral lupus manifested with symptoms of Parkinsonism. The purpose of this case report is to share an uncommon occurrence of cerebral lupus which manifested as Parkinsonism and to highlight the importance of early diagnosis of the condition which is potentially reversibility with prompt treatment [4].
\end{abstract}

(C) 2021 The Author(s)

Published by S. Karger AG, Basel

\section{Introduction}

Cerebral lupus is a severe but potentially curable disease. According to a 3-year prospective analysis by Hanly et al. [1], cerebral lupus events have a detrimental effect on the wellbeing and future quality of life of the patient. The American College of Rheumatology has listed in total 19 syndromes that have been documented in cerebral lupus involving disorders of the central, peripheral, and autonomic nervous systems [2]. Central nervous system comprises the majority of cases reported to date. It can be subcategorized into focal or diffuse. The focal 
Fig. 1. CT scan of the brain showed bilateral basal ganglia

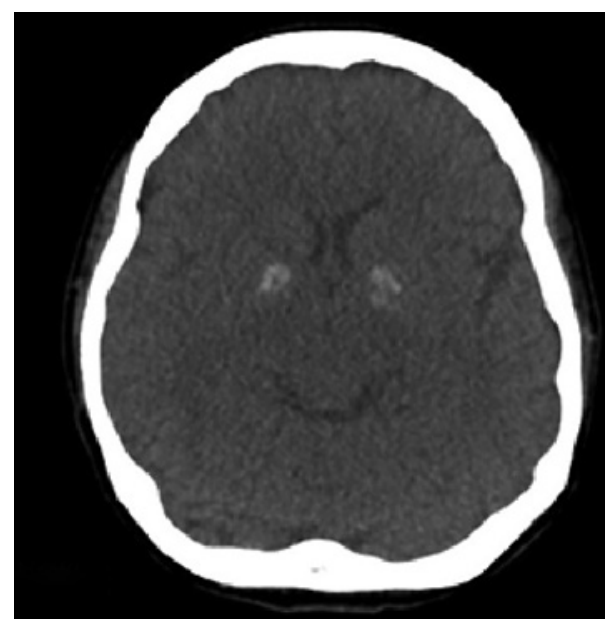
calcifications. CT, computed tomography.

syndromes are mainly neurological, while the diffuse syndromes are mainly psychiatric in nature [3].

With the recent advance in diagnostic imaging technology, the detection of cerebral lupus has increased over the past few decades due to the early recognition of cognitive dysfunction and early manifestation of lupus headaches where no specific pathological changes in the brain that can be seen.

\section{Case Report/Case Presentation}

A 37-year-old woman with an underlying systemic lupus erythematosus (SLE) since 2013 under control with oral prednisolone $20 \mathrm{mg}$ daily, oral hydroxychloroquine $200 \mathrm{mg}$ daily, and oral azathioprine $150 \mathrm{mg}$, presented with sudden onset of headache for the past 3 weeks. The headache was localized over the central parietal region, nonradiating, pulsating in nature, lasted for $9 \mathrm{~h}$, and only partially improved with sleeping. She also noted a gradually decreasing motor function of the lower limbs especially limb movements and reaction to pain stimuli. She has no weakness, no numbness or tingling sensation over her limbs, no drowsiness, no diplopia, no tinnitus, and no urinary incontinence. There was no joint swelling, no photophobia, no fever, or no neck stiffness. There was no oral ulcer, no hair loss, no skin rashes, or other symptoms.

On examinations, she was fully conscious and orientated. There was no expressive or receptive aphasia noted and no hearing impairment. She has an expressionless face, bradykinesia, and a marked cogwheel rigidity over right extremity compared to the left, which suggestive of parkinsonism. Upon walking, she had a stooped posture, and a festinating and hesitant gait with markedly reduced arm swing. There was no resting tremor and no abnormal movement noted.

Laboratory investigations showed hemoglobin $9.9 \mathrm{~g} / \mathrm{dL}$, leucocyte count $12.6 \times 10^{3} / \mathrm{mm}^{3}$, platelet count 270 per $\mu \mathrm{L}$, erythrocyte sedimentation rate $1 \mathrm{~mm} / \mathrm{h}$, C-reactive protein 2.8 $\mathrm{mg} / \mathrm{L}$, antinuclear antibody positive homogenous pattern at 1:640 titer. Her dsDNA, anticardiolipin antibody, lupus anticoagulant, and anti- $\beta 2$-glycoprotein- 1 antibody were negative. Urine test showed $1+$ leucocyte and $1+$ protein. Contrast-enhanced computed tomographic (CT) scan of the brain showed bilateral basal ganglia calcifications (as shown in Fig. 1). There was no leptomeningeal enhancement or cerebral atrophy. Lumbar puncture was performed and showed clear and colorless cerebrospinal fluid with an opening pressure of $10 \mathrm{~cm} \mathrm{H}_{2} \mathrm{O}$. The cerebrospinal fluid analysis was normal and the culture did not grow any organism. Virologic panel test was also carried out and was negative. 
According to SLE Disease Activity Index 2000, her disease activity score at the point of presentation based on the lupus headache was 8 points. This score is high and suggests an active SLE. She was treated as cerebral lupus and was started on intravenous methylprednisolone $500 \mathrm{mg} /$ day for 3 consecutive days. Her headache symptoms improved after treatment. Upon reassessment, her rigidity disappeared on the second day of treatment, and her gait, speech, and upper limb movement also showed marked improvement. She never started on any dopaminergic medication. She was discharged well with oral prednisolone $25 \mathrm{mg}$ daily for 1 month and an appointment with rheumatologist for a review.

\section{Discussion/Conclusion}

It is irrefutable that this patient has clinical features of Parkinsonism. Her initial presenting complaint was persistent headache, where she was admitted for further evaluation and treatment. The cardinal clinical diagnosis made on initial assessment was cerebral lupus. An urgent contrast-enhance CT scan of the brain was done to rule out stroke or any other brain pathology. A thorough medication history of patient was taken in order to rule out the possibility of drug-induced Parkinsonism. Lumbar puncture was also performed and investigated for any infection of the central nervous system, which was negative in this case. As the patient has no previous history of encephalitis and demonstrated a rapid improvement of her parkinsonism features after steroid treatment, we can certainly rule out infective causes such as post-encephalitic parkinsonism.

The contrast-enhanced CT scan of the brain of the patient showed bilateral basal ganglia calcification. There were several similar case reports that showed the same reporting from CT or magnetic resonance imaging scan of the brain, which is the underlying pathology of Parkinsonism [5, 6]. In general, the basic pathological lesion of many neurological changes is the vasculitis leading to hemorrhages, microinfarcts, perivascular fibrosis, and encephalomalacia in the nervous system. The autopsy finding of encephalomalacia in the basal ganglia in patient with cogwheel rigidity supports our concept that parkinsonism can be initiated by vasculitis in the corpus striatum involving globus pallidus and substantia nigra [5]. As the neurotransmitter dopamine supply is cut off when the striatum is diseased, the neuronal interactions in the cortex that co-ordinate and modulate the subsequent movements are affected [6]. In our case, the treatment of immunosuppression with steroid was thought to be in time and thus reversed the inflammatory process and prevented irreversible damages such as microinfracts and fibrosis. We expect that if the treatment was delayed, the neurological symptoms in this patient would be permanent. The limitation in our case is lack of Magnetic Resonance Imaging scan of brain to confirm the radiological findings. During our follow-up with this patient, the patient did not have any neurological deficits. We expect that the outcome for this patient would be good based on the fact that she responded to immunosuppression during the acute onset of cerebral lupus and that with subsequent therapy with oral prednisolone she was asymptomatic.

In conclusion, parkinsonism is an uncommon neurological manifestation of cerebral lupus which is reversible with corticosteroid and immunosuppressive treatment. Therefore, early recognition of the condition is important as it can improve the prognosis and quality of life of the patient in future.

\section{Acknowledgements}

The author would like to thank the Director General of Ministry of Health of Malaysia for his permission to publish this article.

\section{Karger'}




\section{Statement of Ethics}

Written informed consent was obtained from the patient for publication of this case report and any accompanying images. The paper is exempt from Ethical Committee approval because it only involves a single patient and only requires informed consent from the patient.

\section{Conflict of Interest Statement}

The authors have no conflicts of interest to declare.

\section{Funding Sources}

This publication did not receive any funding support as it is just a case report.

\section{Author Contributions}

Chee Keong Chang contributed to writing and editing. Malehah Mohd Noh contributed to supervisor and final editing. Constance Liew Sat Lin contributed to data collection. Alvin Oliver Payus contributed to writing, review, and final editing.

\section{Data Availability Statement}

All data generated or analyzed during this study are included in this article. Further enquiries can be directed to the corresponding author.

\section{References}

1 Hanly JG, Urowitz MB, Su L, Bae SC, Gordon C, Wallace DJ, et al. Prospective analysis of neuropsychiatric events in an international disease inception cohort of patients with systemic lupus erythematosus. Ann Rheum Dis. 2010 Mar 1;69(3):529-35.

2 Liang MH, Corzillius M, Bae SC, Lew RA, Fortin PR, Gordon C, et al. The American College of Rheumatology nomenclature and case definitions for neuropsychiatric lupus syndromes. Arthritis Rheum. 1999 Apr;42(4): 599-608.

3 Unterman A, Nolte JE, Boaz M, Abady M, Shoenfeld Y, Zandman-Goddard G. Neuropsychiatric syndromes in systemic lupus erythematosus: a meta-analysis. Semin Arthritis Rheum. 2011 Aug 1;41(1):1-11.

4 Kwong KL, Chu R, Wong SN. Parkinsonism as unusual neurological complication in childhood systemic lupus erythematosus. Lupus. 2000 Jul; $9(6): 474-7$.

5 Lee PH, Joo US, Bang OY, Seo CH. Basal ganglia hyperperfusion in a patient with systemic lupus erythematosusrelated parkinsonism. Neurology. 2004 Jul 27;63(2):395-6.

6 Khubchandani RP, Viswanathan V, Desai J. Unusual neurologic manifestations (I): Parkinsonism in juvenile SLE. Lupus. 2007 Aug;16(8):572-5.

\section{Karger' ${ }^{\prime}$}

